\title{
Microarray-based genome investigation: molecular karyotyping or segmental aneuploidy profiling?
}

\author{
European Journal of Human Genetics (2006) 14, 262-265. doi:10.1038/sj.ejhg.5201553; published online 4 January 2006
}

In several recent publications the term 'molecular karyotyping' was adopted to describe the use of microarrays for detecting genomic imbalances, in analogy to conventional karyotyping based on analysis of metaphase chromosomes. We argue that this term is a misnomer, because microarraybased techniques do not reveal any details about chromosome number or structure. In addition, the term has already occupied a place in molecular genetics, referring to the separation of chromosomes based on their physical properties, for example, by pulsed-field gel electrophoresis or flow-sorting. As an alternative to 'molecular karyotyping' we propose the more accurate term 'segmental aneuploidy profiling (SAP)'.

In several recent publications, ${ }^{1-4}$ detection of chromosomal imbalances by hybridization of genomic DNA to microarrays has been described as 'molecular karyotyping' (Table 1). Clearly, the data obtained by microarray-based methods extend and complement those of conventional molecular cytogenetic investigations based on metaphase chromosome-banding techniques and fluorescence in situ hybridization (FISH). Here, we argue that 'molecular karyotyping' is not an adequate description in this context.

Both karyotyping and microarray-based comparative genomic hybridization are methods to identify gains and losses throughout the genome in order to provide an explanation for an abnormal phenotype. The resolving power of karyotyping is limited by the representation of the genome in differentially stained chromosome bands. In other words, a gain or loss of genomic DNA can be detected only if the chromosomal banding pattern is affected by the genomic imbalance. By definition, this limits the resolving power of karyotyping to several megabases. In contrast, the resolution provided by microarray-based methods may still be beyond our imagination, reaching the kilobase level when several hundred thousands of SNP markers are used. Microarrays can provide precise information regarding the location, and hence, the gene content, of genomic imbalances. When it comes to making genotype-phenotype correlations in everyday clinical genetic practice and research, microarray-based methods represent a giant leap forward compared to conventional karyotyping. However, may we adopt the name 'molecular karyotyping' for this new methodology?
The term 'molecular karyotyping' has been proposed by Vermeesch et $a l^{1}$ to describe identification of genomewide chromosomal aberrations by microarray comparative genomic hybridization (CGH). In order to understand why the term 'molecular karyotyping' is inadequate in this context, we first need to acknowledge what, traditionally, has been implied by the term 'karyotype' (with 'karyo-' from the Greek word 'karyon', meaning 'nut' or 'kernel'). It was first used in 1924 by Levitsky, ${ }^{5}$ a professor of botany at the University of Kiew in Russia. The karyotype describes 'the particular chromosome complement of an individual or of a related group of individuals, as defined both by the number and morphology of the chromosomes'. ${ }^{6}$ The number and morphology of the chromosomes are most easily studied by observing metaphase plates. The normal human karyotype was established by Tjio and Levan, and independently, by Ford and Hamerton, in 1956 by studying metaphase plates from embryonic lung fibroblasts, and spermatogonia, respectively. ${ }^{7}$ The development of lymphocyte culturing and chromosome banding techniques for routine use in the clinical cytogenetics laboratory allows metaphase chromosome structure to be routinely studied at a level of $\sim 700$ bands, and sophisticated nomenclature has been developed to describe any abnormal karyotype. ${ }^{7}$

The term 'molecular karyotyping' proposed by Vermeesch et $a l^{1}$ is a misnomer because of two intrinsic reasons. First, microarray-based methods do not reveal the number, size or structure (ie 'morphology' cf Levitsky5) of the chromosomes involved. For example, a segmental duplication detected by microarray-based techniques could have been caused by a variety of structural chromosome abnormalities (Table 2a). Conversely, individuals who harbour a 'normal molecular karyotype', may carry a variety of balanced, but abnormal karyotypes (Table $2 \mathrm{~b}$ ). This applies, for example, to patients suffering from mental retardation and multiple congenital abnormalities who have a de novo, reciprocal translocation recognizable at metaphase, with one of the chromosomal breaks disrupting a positional candidate gene for the phenotype. In the examples given in Table 3, cloning and DNA sequence analysis of both translocation breakpoints proved that the translocation was truly reciprocal, and that only one of the 
Table 1 Recent publications describing the clinical use of microarrays for aneuploidy detection as 'molecular karyotyping'

\begin{tabular}{lc}
\hline Title & Microarray platform \\
\hline Molecular karyotyping: array CGH quality criteria for constitutional genetic diagnosis & 3527 BAC/PAC \\
Molecular karyotyping using an SNP array for genomewide genotyping & 10000 SNP \\
Microarray analysis of cell-free fetal DNA in amniotic fluid: a prenatal molecular karyotype & 287 targets \\
Molecular karyotyping in human constitutional cytogenetics & None - review article \\
\hline
\end{tabular}

breakpoints interrupted a protein coding DNA sequence. Such balanced chromosomal rearrangements, with severe consequences for the patient but of tremendous benefit for the purpose of gene identification, escape detection by whole-genome hybridization to microarrays, even when high-resolution platforms are used. The breakpoints of such rearrangements can be identified using microarrays by hybridization of probes that have been prepared from the flow-sorted, aberrant chromosomes, a technique called 'array painting' ${ }^{8}$ Prior to this, the rearrangements need to be identified by conventional karyotyping.

There is a second intrinsic distinction between both approaches. The karyotype is determined by studying metaphases. Therefore, it is essentially a cell-by-cell approach, enabling, for example, identification of chromosomal mosaicism if two cell lines coexist in an individual, each with its own particular karyotype. In contrast, the DNA-samples used for microarray-based genome analysis represent many cells from an individual. Chromosomal mosaicism can be detected by BAC-based microarray-CGH, but only if the least abundant cell type is present at a frequency of at least $\sim 20 \%{ }^{1}$ This percentage may drop as techniques are improved, but irrespectively, a much higher sensitivity for detecting mosaicism can be achieved by studying metaphases. For example, the finding of two metaphases with a supernumerary chromosome, each in an independent culture, is conventionally taken among clinical cytogeneticists as sufficient evidence for chromosomal mosaicism.

In addition to these two intrinsic arguments, there are also historical reasons for avoiding the use of the term 'molecular karyotyping' as an analogy to metaphase-based karyotyping. The term 'molecular karyotyping' first appeared in the literature during the eighties of the 20th century, when pulsed-field gel electrophoresis (PFGE) enabled physical separation of the megabase-sized interphase, not metaphase, chromosomes of human parasites, such as Leishmania major, ${ }^{9}$ Trypanosoma cruzi, $^{10}$ and Plasmodium falciparum. ${ }^{11}$ The term is adequately used here because chromosomes are separated, allowing their number and physical size (ie 'morphology' cf Levitsky') to be determined. Fractionation of chromosomes by PFGE in these species yields an ordered arrangement of the chromosomes. This truly represents a 'molecular karyotype'. It can be used to assign cloned fragments of genomic DNA or cDNAs to a specific chromosome by genomic
Table 2 (a) Abnormal chromosome structures detected as a segmental duplication by microarray-based methods and (b) Balanced, abnormal karyotypes not detectable by microarray-based methods

\section{(a) \\ Chromosomal abnormality}

Direct/inverted duplication

Direct/inverted within-arm insertion

Direct/inverted between-arm insertion

Direct/inverted insertion into the other homologous chromosome

Direct/inverted insertion into a nonhomologous chromosome Unbalanced form of reciprocal subtelomeric translocation Small supernumerary chromosome

(b)

\begin{tabular}{lc} 
Chromosomal abnormality & $\begin{array}{c}\text { Chromosome } \\
\text { number }\end{array}$ \\
\hline Reciprocal translocation & 46 \\
Whole-arm exchange & 46 \\
Inversion (para- and pericentric) & 46 \\
Insertion originating from three 3-break event & 46 \\
Reciprocal exchange of interstitial segments & 46 \\
(4-breaks) & 45 or 46 \\
Robertsonian translocation (ie centric fusion) & 47 \\
Centric fission & 47 \\
\hline
\end{tabular}

Southern blot hybridization, which is in perfect analogy to gene mapping by FISH on metaphase chromosomes. This method is being used until the present day, for example in the Trypanosoma cruzi Genome Project for constructing physical maps of the chromosomes of the reference clone CL Brener. ${ }^{12}$ In addition, molecular karyotyping of these parasites allowed studying the evolution of their genomes, again in analogy to studies of genome evolution by comparative cytogenetic investigations in species that more easily yield metaphase chromosomes. Thus, the term 'molecular karyotyping' describes a well-defined concept in molecular genetics. In humans, where chromosomes are too large for fractionation by PFGE, the closest equivalent to veritable 'molecular karyotyping' was, again, during the eighties of the 20th century, arrived at by flow cytometry of metaphase chromosomes, generating a 'flow karyotype'. ${ }^{13-15}$ Hybridization of DNA-probes to flow-sorted human metaphase chromosomes spotted on a 
Table 3 Selected examples of de novo, reciprocal translocations with one of the breakpoints disrupting a positional candidate gene for the clinical phenotype

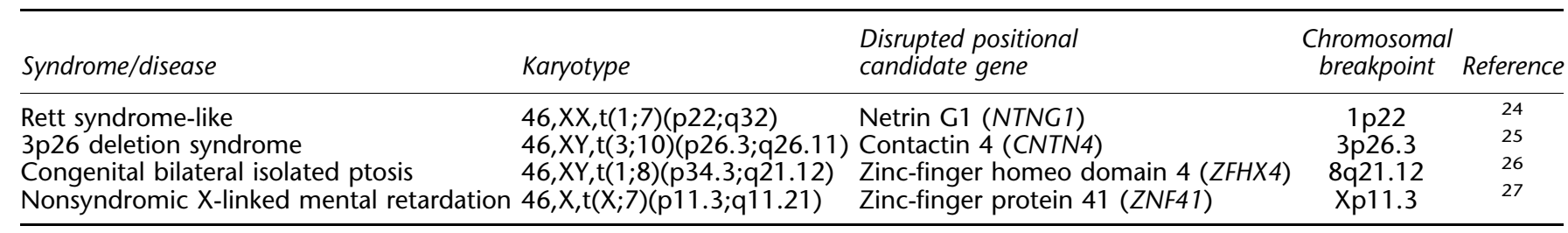

nitrocellulose filter has been used successfully for gene mapping, ${ }^{16}$ in perfect analogy to gene mapping by metaphase FISH.

We conclude that metaphase cytogenetics and microarray-based copy number quantification are distinct, but complementary approaches, yielding data that provide clinically meaningful information only when combined. The result of a microarray analysis provides, in essence, a profile of the segmental aneuploidies present in a genome. The resolving power and precision of aneuploidy detection using microarrays is unattainable by chromosome banding. Only studying metaphase chromosomes, using chromosome-banding techniques and FISH, can identify the structural basis and, hence, the mechanism of origin of the chromosomal aneuploidy. Elucidation of the mechanism of origin is crucial for genetic counselling of parents of a child with a genomic imbalance who wish to be informed about the recurrence risk.

As an alternative to 'molecular karyotyping' to describe microarray-based genome investigation, we propose the more precise term 'segmental aneuploidy profiling' (SAP), in accordance with Lindsley et al. ${ }^{17}$ By adopting this term, we need to extend the meaning of the term 'aneuploidy' in order to accommodate the recent identification of numerous segmental aneuplodies in healthy individuals. ${ }^{18-22}$ In a classical sense, aneuploidy refers to chromosomes and chromosome segments that are absent from, or exist in addition to, a basic chromosome set, ${ }^{6}$ and that in most cases have been recognized because of their association with an abnormal phenotype. The classical meaning of the term relies on the existence of a 'basic chromosome set' (a perfectly euploid genome) in healthy individuals. As shown recently, numerous segmental aneuploidies (also termed 'copy number polymorphisms' or 'large-scale copy number variations') are shared between unrelated, apparently healthy humans. ${ }^{18-20}$ These genomic variations have also been found in commonly used inbred laboratory mouse strains. ${ }^{21-22}$ The regions of copy number variation thus identified may be as large as several hundred kilobases of DNA, and many contain protein coding genes. Whereas the phenotypic relevance of these genomic imbalances is unknown, it is clear that a perfectly euploid genome may not exist in mice and men. Of course, a perfectly euploid genome would be the ideal reference sample for detecting segmental aneuploidies by array-CGH. For this purpose we have created a synthetic diploid human genome by combining equal amounts of genomic DNA from 50 healthy subjects of the same sex. ${ }^{23}$ In the absence of an euploid genome, the classical meaning of 'aneuploidy' requires reconsideration. We propose that the term 'segmental aneuploidy' refers to all deviations from an ideal, perfectly diploid chromosome complement as revealed by hybridization of genomic DNA to microarrays. These include both copy number changes of apparent clinical relevance (as defined by their occurrence in one particular patient), and those that are, apparently, not clinically relevant (as defined by their occurrence in multiple, unrelated, healthy subjects). This is an appropriate extension of the classical meaning of the term aneuploidy, allowing to accommodate data generated by microarraybased genomic hybridization.

Ron Hochstenbach ${ }^{*}{ }^{1}$, Hans Kristian Ploos van Amstel ${ }^{1}$ and Martin Poot $^{1}$

${ }^{1}$ Department of Biomedical Genetics, University Medical Centre Utrecht, PO Box 85090, 3508 AB Utrecht, The Netherlands

*Correspondence: $R$ Hochstenbach, Department of Biomedical Genetics, University Medical Centre Utrecht, PO Box 85090, Room KC.04.084.2, 3508 AB Utrecht, The Netherlands. Tel: + 3130250 3800; Fax: + 31302503801 ; E-mail: p.f.r.hochstenbach@dmg.azu.nl

\section{References}

1 Vermeesch JR, Melotte C, Froyen G et al: Molecular karyotyping: array CGH quality criteria for constitutional genetic diagnosis. J Histochem Cytochem 2005; 53: 413-422.

2 Rauch A, Rüschendorf F, Huang J et al: Molecular karyotyping using an SNP array for genomewide genotyping. J Med Genet 2004; 41: 916-922.

3 Larabee PB, Johnson KL, Pestova E et al: Microarray analysis of cell-free fetal DNA in amniotic fluid: a prenatal molecular karyotype. Am J Hum Genet 2004; 75: 485-491.

4 Sanlaville D, Lapierre J-M, Turleau C et al: Molecular karyotyping in human constitutional cytogenetics. Eur J Med Genet 2005; 48: $214-231$.

5 Levitsky GA: Materielle Grundlagen der Vererbung (russ). Kiew: Staatsverlag, 1924.

6 Rieger R, Michaelis A, Green MM: Glossary of Genetics - Classical and Molecular. Berlin: Springer Verlag, 5th edn., 1991. 
7 ISCN (2005): An International System for Human Cytogenetic Nomenclature. Shaffer Lg, Tommer N (eds): Basel: Karger, 2005.

8 Fiegler H, Gribble SM, Burford DC et al: Array painting: a method for the rapid analysis of aberrant chromosomes using DNA microarrays. J Med Genet 2004; 40: 664-670.

9 Spithill TW, Samaras N: The molecular karyotype of Leishmania major and mapping of alpha and beta tubulin gene families to multiple unlinked chromosomal loci. Nucleic Acids Res 1985; 13: 4155-4169.

10 Engman DM, Reddy LV, Donelson JE, Kirchhoff LV: Trypanosoma cruzi exhibits inter- and intra-strain heterogeneity in molecular karyotype and chromosomal gene location. Mol Biochem Parasitol 1987; 15: 115-123.

11 Kemp DJ, Thompson JK, Walliker D, Corcoran LM: Molecular karyotype of Plasmodium falciparum: conserved linkage groups and expendable histidine-rich protein genes. Proc Natl Acad Sci USA 1987; 84: 7672-7676.

12 Porcile PE, Santos MRM, Souza RT et al: A refined molecular karyotype for the reference strain of the Trypanosoma cruzi genome project (clone CL Brener) by assignment of chromosome markers. Gene 2003; 308: 53-65.

13 Young BD, Ferguson-Smith MA, Sillar R, Boyd E: High-resolution analysis of human peripheral lymphocyte chromosomes by flow cytometry. Proc Natl Acad Sci USA 1981; 78: 7727-7731.

14 Bernheim A, Metezeau P, Guellaën G, Fellous M, Golberg ME, Berger R: Direct hybridization of sorted human chromosomes: localization of the Y chromosome on the flow karyotype. Proc Natl Acad Sci USA 1983; 80: 7571-7575.

15 Trask B, van den Engh G, Mayall B, Gray JW: Chromosome heteromorphism quantified by high-resolution bivariate flow karyotyping. Am J Hum Genet 1989; 45: 739-752.

16 Collard JG, de Boer PA, Janssen JW, Schijven JF, de Jong B: Gene mapping by chromosome spot hybridization. Cytometry 1985; 6 : $179-185$
17 Lindsley DL, Sandler L, Baker BS et al: Segmental aneuploidy and the genetic gross structure of the Drosophila genome. Genetics 1972; 71: 157-184

18 Iafrate AJ, Feuk L, Rivera MN et al: Detection of large-scale variation in the human genome. Nat Genet 2004; 36: 949-951.

19 Sebat J, Lakshmi B, Troge J et al: Large-scale copy number polymorphism in the human genome. Science 2004; 305: 525-528.

20 Sharp AJ, Locke DP, McGrath SD et al: Segmental duplications and copy-number variation in the human genome. Am J Hum Genet 2005; 77: 78-88.

$21 \mathrm{Li} \mathrm{J}$, Jiang T, Mao J-H et al: Genomic segmental polymorphisms in inbred mouse strains. Nature Genet 2004; 36: 952-954.

22 Adams DJ, Dermitzakis ET, Cox T et al: Complex haplotypes, copy number polymorphisms and coding variation in two recently divergent mouse strains. Nature Genet 2005; 37: 532-536.

23 Lichtenbelt KD, Hochstenbach R, van Dam MW, Eleveld MJ, Poot M, Beemer FA: Supernumerary ring chromosome 7 mosaicism: case report, investigation of the gene content, and delineation of the phenotype. Am J Med Genet A 2005; 132: 93-100.

24 Borg I, Freude K, Kubart S et al: Disruption of Netrin G1 by a balanced chromosome translocation in a girl with Rett syndrome. Eur I Hum Genet 2005; 13: 921-927.

25 Fernandez T, Morgan T, Davis N et al: Disruption of contactin 4 (CNTN4) results in developmental delay and other features of $3 p$ deletion syndrome. Am J Hum Genet 2004; 74: 1286-1293.

26 McMullan TFW, Crolla JA, Gregory SG et al: A candidate gene for congenital bilateral isolated ptosis identified by molecular analysis of a de novo balanced translocation. Hum Genet 2002; 110: $244-250$.

27 Shoichet SA, Hoffmann K, Menzel C et al: Mutations in the ZNF41 gene are associated with cognitive deficits: identification of a new candidate for X-linked mental retardation. Am J Hum Genet 2003; 73: 1341-1354 